\title{
The conservation status of the world's reptiles
}

Monika Böhm ${ }^{\mathrm{a}, *}$, Ben Collen ${ }^{\mathrm{a}}$, Jonathan E.M. Baillie ${ }^{\mathrm{b}}$, Philip Bowles ${ }^{\mathrm{c}}$, Janice Chanson ${ }^{\mathrm{d}, \mathrm{e}}$, Neil Cox ${ }^{\mathrm{c}, \mathrm{d}}$, Geoffrey Hammerson ${ }^{f}$, Michael Hoffmann ${ }^{g}$, Suzanne R. Livingstone ${ }^{h}$, Mala Ram ${ }^{a}$, Anders G.J. Rhodin ${ }^{\mathrm{i}}$, Simon N. Stuart ${ }^{\mathrm{j}, \mathrm{k}, \mathrm{l}, \mathrm{m}, \mathrm{n}}$, Peter Paul van Dijk ${ }^{1}$, Bruce E. Young ${ }^{\mathrm{o}}$, Leticia E. Afuang ${ }^{\mathrm{p}}$, Aram Aghasyan ${ }^{\mathrm{q}}$, Andrés García $^{\mathrm{r}}$, César Aguilar ${ }^{\mathrm{s}}$, Rastko Ajtic ${ }^{\mathrm{t}}$, Ferdi Akarsu ${ }^{\mathrm{u}}$, Laura R.V. Alencar ${ }^{\mathrm{v}}$, Allen Allison ${ }^{\mathrm{w}}$, Natalia Ananjeva ${ }^{\mathrm{x}}$, Steve Anderson ${ }^{\mathrm{y}}$, Claes Andrén ${ }^{\mathrm{z}}$, Daniel Ariano-Sánchez ${ }^{\mathrm{aa}}$, Juan Camilo Arredondo ${ }^{\mathrm{ab}}$, Mark Auliya ${ }^{\mathrm{ac}}$, Christopher C. Austin ${ }^{\text {ad }}$, Aziz Avci ${ }^{\text {ae }}$, Patrick J. Baker ${ }^{\text {af,ag }}$, André F. Barreto-Lima ${ }^{\text {ah }}$, César L. Barrio-Amorós ${ }^{\text {ai }}$, Dhruvayothi Basu $^{\text {aj }}$, Michael F. Bates ${ }^{\text {ak }}$, Alexandre Batistella ${ }^{\text {al }}$, Aaron Bauer ${ }^{a m}$, Daniel Bennett ${ }^{\text {an }}$, Wolfgang Böhme $^{\text {ao }}$, Don Broadley ${ }^{\text {ap }}$, Rafe Brown ${ }^{\text {aq }}$, Joseph Burgess ${ }^{\text {ar }}$, Ashok Captain ${ }^{\text {as }}$, Santiago Carreira ${ }^{\text {at }}$, Maria del Rosario Castañeda $^{\text {au }}$, Fernando Castro ${ }^{\text {av }}$, Alessandro Catenazzi ${ }^{\text {aw }}$, José R. Cedeño-Vázquez ${ }^{\text {ax }}$, David G. Chapple $^{\text {ay,az }}$, Marc Cheylan ${ }^{\text {ba }}$, Diego F. Cisneros-Heredia ${ }^{\text {bb }}$, Dan Cogalniceanu ${ }^{\text {bc }}$, Hal Cogger ${ }^{\text {bd }}$, Claudia Corti $^{\text {be }}$, Gabriel C. Costa ${ }^{\text {bf }}$, Patrick J. Couper ${ }^{\text {bg }}$, Tony Courtney ${ }^{\text {bh }}$, Jelka Crnobrnja-Isailovic ${ }^{\text {bi }}$, Pierre-André Crochet $^{\text {ba }}$, Brian Crother ${ }^{\text {bj }}$, Felix Cruz ${ }^{\text {bk }}$, Jennifer C. Daltry ${ }^{\text {bl }}$, R.J. Ranjit Daniels ${ }^{\text {bm }}$, Indraneil Das ${ }^{\text {bn }}$, Anslem de Silva ${ }^{\text {bo,bp }}$, Arvin C. Diesmos ${ }^{\text {bq }}$, Lutz Dirksen ${ }^{\text {br }}$, Tiffany M. Doan ${ }^{\text {bs }}$, C. Kenneth Dodd Jr. ${ }^{\text {bt }}$, J. Sean Doody ${ }^{\text {ay }}$, Michael E. Dorcas ${ }^{\text {bu }}$, Jose Duarte de Barros Filho ${ }^{\text {bv }}$, Vincent T. Egan ${ }^{\text {bw }}$, El Hassan El Mouden ${ }^{\text {bx }}$, Dirk Embert $^{\text {by }}$, Robert E. Espinoza ${ }^{\text {bz }}$, Alejandro Fallabrino ${ }^{\mathrm{ca}}$, Xie Feng ${ }^{\mathrm{cb}}$, Zhao-Jun Feng ${ }^{\mathrm{cc}}$, Lee Fitzgerald ${ }^{\mathrm{cd}}$, Oscar Flores-Villela ${ }^{\mathrm{ce}}$, Frederico G.R. França ${ }^{\mathrm{cf}}$, Darrell Frost ${ }^{\mathrm{cg}}$, Hector Gadsden ${ }^{\mathrm{ch}}$, Tony Gamble ${ }^{\mathrm{ci}}$, S.R. Ganesh ${ }^{\mathrm{cj}}$, Miguel A. Garcia ck, Juan E. García-Pérez ${ }^{\mathrm{cl}}$, Joey Gatus ${ }^{\mathrm{cm}}$, Maren Gaulke ${ }^{\mathrm{cn}}$, Philippe Geniez ${ }^{\mathrm{co}}$, Arthur Georges ${ }^{\mathrm{cp}}$, Justin Gerlach ${ }^{\mathrm{cq}}$, Stephen Goldberg ${ }^{\mathrm{cr}}$, Juan-Carlos T. Gonzalez ${ }^{\mathrm{p}, \mathrm{cs}}$, David J. Gower ${ }^{\mathrm{ct}}$, Tandora Grant $^{\mathrm{cu}}$, Eli Greenbaum ${ }^{\mathrm{cv}}$, Cristina Grieco ${ }^{\mathrm{cw}}$, Peng Guo ${ }^{\mathrm{cx}}$, Alison M. Hamilton ${ }^{\mathrm{cy}}$, Kelly Hare ${ }^{\mathrm{cz}}$, S. Blair Hedges $^{\text {da }}$, Neil Heideman ${ }^{\mathrm{db}}$, Craig Hilton-Taylor ${ }^{\mathrm{dc}}$, Rod Hitchmough ${ }^{\mathrm{dd}}$, Bradford Hollingsworth ${ }^{\text {de }}$, Mark Hutchinson $^{\mathrm{df}}$, Ivan Ineich ${ }^{\mathrm{dg}}$, John Iverson ${ }^{\mathrm{dh}}$, Fabian M. Jaksic ${ }^{\mathrm{di}}$, Richard Jenkins ${ }^{\mathrm{dj}, \mathrm{dk}, \mathrm{dl}}{ }^{\text {, Ulrich Joger }}{ }^{\mathrm{dm}}$, Reizl

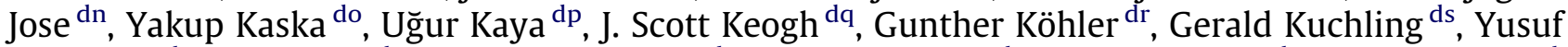
Kumlutaş ${ }^{\mathrm{dt}}$, Axel Kwet ${ }^{\mathrm{du}}$, Enrique La Marca ${ }^{\mathrm{dv}}$, William Lamar ${ }^{\mathrm{dw}}$, Amanda Lane ${ }^{\mathrm{dx}}$, Bjorn Lardner ${ }^{\mathrm{dy}}$, Craig Latta $^{\mathrm{dz}}$, Gabrielle Latta ${ }^{\mathrm{dz}}$, Michael Lau ${ }^{\mathrm{ea}}$, Pablo Lavin ${ }^{\mathrm{eb}}$, Dwight Lawson ${ }^{\mathrm{ec}}$, Matthew LeBreton ${ }^{\text {ed }}$, Edgar Lehr $^{\text {ee }}$, Duncan Limpus ef ${ }^{\text {encola Lipczynski }}{ }^{\text {eg }}$, Aaron S. Lobo ${ }^{\text {eh }}$, Marco A. López-Luna ${ }^{\text {ei }}$, Luca Luiselli ${ }^{\text {ej }}$, Vimoksalehi Lukoschek ${ }^{\text {ek,el }}$, Mikael Lundberg ${ }^{\mathrm{em}}$, Petros Lymberakis ${ }^{\mathrm{en}}$, Robert Macey ${ }^{\mathrm{eo}}$, William E. Magnusson $^{\text {ep }}$, D. Luke Mahler ${ }^{\text {eq }}$, Anita Malhotra ${ }^{\text {er }}$, Jean Mariaux ${ }^{\text {es }}$, Bryan Maritz ${ }^{\text {et }}$, Otavio A.V. Marques ${ }^{\text {eu }}$, Rafael Márquez $^{\mathrm{ev}}$, Marcio Martins ${ }^{\mathrm{v}}$, Gavin Masterson ${ }^{\mathrm{et}}$, José A. Mateo ${ }^{\mathrm{ew}}$, Rosamma Mathew ${ }^{\mathrm{ex}}$, Nixon Mathews ${ }^{\mathrm{ey}}$, Gregory Mayer ${ }^{\mathrm{ez}}$, James R. McCranie ${ }^{\mathrm{fa}}$, G. John Measey ${ }^{\mathrm{fb}}$, Fernando Mendoza-Quijano ${ }^{\mathrm{fc}}$, Michele Menegon ${ }^{\mathrm{fd}}$, Sébastien Métrailler ${ }^{\mathrm{fe}}$, David A. Milton ${ }^{\mathrm{ff}}$, Chad Montgomery ${ }^{\mathrm{fg}}$, Sérgio A.A. Morato ${ }^{\mathrm{fh}}$, Tami Mott ${ }^{\mathrm{fi}}$, Antonio Muñoz-Alonso ${ }^{\mathrm{fj}}$, John Murphy ${ }^{\mathrm{fk}}$, Truong Q. Nguyen ${ }^{\text {ao,fl }}$, Göran Nilson ${ }^{\text {fm }}$, Cristiano Nogueira $^{\text {fn }}$, Herman Núñez ${ }^{\text {fo }}$, Nikolai Orlov ${ }^{\mathrm{x}}$, Hidetoshi Ota ${ }^{\mathrm{fp}}$, José Ottenwalder ${ }^{\mathrm{fq}}$, Theodore Papenfuss ${ }^{\mathrm{fr}}$, Stesha Pasachnik ${ }^{\mathrm{fs}}$, Paulo Passos ${ }^{\mathrm{ft}}$, Olivier S.G. Pauwels ${ }^{\mathrm{fu}}$, Néstor Pérez-Buitrago ${ }^{\mathrm{fv}}$, Valentín PérezMellado $^{\text {fw }}$, Eric R. Pianka ${ }^{\text {fx }}$, Juan Pleguezuelos ${ }^{\text {fy }}$, Caroline Pollock ${ }^{\text {dc }}$, Paulino Ponce-Campos ${ }^{\text {fz }}$, Robert Powell $^{\text {ga }}$, Fabio Pupin ${ }^{\text {fd }}$, Gustavo E. Quintero Díaz ${ }^{\text {gb }}$, Raju Radder ${ }^{\text {gc }}$, Jan Ramer ${ }^{\text {gd }}$, Arne R. Rasmussen ${ }^{\text {ge }}$, Chris Raxworthy ${ }^{\mathrm{cg}}$, Robert Reynolds ${ }^{\text {gf }}$, Nadia Richman ${ }^{\mathrm{a}}$, Edmund L. Rico ${ }^{\text {gg }}$, Elisa Riservato ${ }^{\text {gh }}$, Gilson Rivas ${ }^{\text {gi }}$, Pedro L.B. da Rocha ${ }^{\text {gj, Mark-Oliver Rödel }}{ }^{\text {gk }}$, Lourdes Rodríguez Schettino ${ }^{\text {gl }}$, Willem M. Roosenburg ${ }^{\text {gm }}$, James P. Ross ${ }^{\text {bt,gn }}$, Riyad Sadek ${ }^{\text {go }}$, Kate Sanders ${ }^{\text {gp }}$, Georgina Santos-Barrera ${ }^{\text {gq }}$, Hermann H. Schleich ${ }^{\text {gr }}$, Benedikt R. Schmidt ${ }^{\mathrm{gs}, \mathrm{gt}}$, Andreas Schmitz ${ }^{\mathrm{gu}}$, Mozafar Sharifi ${ }^{\mathrm{gv}}$, Glenn Shea ${ }^{\mathrm{dx}}$, Hai-Tao Shi ${ }^{\text {gw }}$, Richard Shine $^{\mathrm{gc}}$, Roberto Sindaco ${ }^{\mathrm{cw}}$, Tahar Slimani ${ }^{\mathrm{bx}}$, Ruchira Somaweera ${ }^{\mathrm{gc}}$, Steve Spawls ${ }^{\mathrm{gx}}$, Peter Stafford ${ }^{\mathrm{ct}}$, Rob 